\title{
Routine Iron Supplementation during Pregnancy: Its Reflection on Iron and Oxidative Status in a Cohort of Pregnant Women in Kinshasa, DR Congo
}

\author{
Andy Muela Mbangama*, Barthélémy Tandu-Umba, Roger Mwimba Mbungu \\ Department of Obstetrics and Gynecology, University Clinics of Kinshasa, Kinshasa, DR Congo \\ Email:*dymuela@yahoo.fr
}

How to cite this paper: Mbangama, A.M., Tandu-Umba, B. and Mbungu, R.M. (2019) Routine Iron Supplementation during Pregnancy: Its Reflection on Iron and Oxidative Status in a Cohort of Pregnant Women in Kinshasa, DR Congo. Open Journal of Obstetrics and Gynecology, 9, 98-115. https://doi.org/10.4236/ojog.2019.91011

Received: December 24, 2018

Accepted: January 22, 2019

Published: January 25, 2019

Copyright $\odot 2019$ by author(s) and Scientific Research Publishing Inc. This work is licensed under the Creative Commons Attribution International License (CC BY 4.0).

http://creativecommons.org/licenses/by/4.0/

\begin{abstract}
Background: Due to maternal and fetal physiologic demand, the rate along with amplitude of anemia is expected to rise over pregnancy. The iron and oxidative status also are expected to vary accordingly. It is thus assumed that iron supplementation will somewhat modify the profile observed in markers of iron and oxidative status of our series. Objectives: We aimed to measure variations of indices of iron and oxidative status of iron supplemented women over pregnancy. Methods: This is a prospective observational cohort of 74 pregnant women with singleton pregnancy whose baseline iron and oxidative status have been recently assessed at University Clinics of Kinshasa, DR Congo. Women with anemia $(\mathrm{Hb}<10 \mathrm{~g} \%)$ took iron for curative purposes $(320$ $\mathrm{mg}$ /day of ferric ammonium citrate) while the others received preventive iron supplementation $(160 \mathrm{mg} /$ day). Iron was associated with $15 \mathrm{mg}$ folate/day. Nutritional iron intake was measured through a 24 -hour recall using a questionnaire of common foods, including stuffs and habits likely to impede iron absorption. Biological parameters of iron and oxidative status included hemoglobin, hematocrit, ferritin, serum iron, transferrin, superoxide dismutase or SOD, uric acid, oxidized LDL and blood glucose. For statistical calculations we used t-test, chi-square test, ANOVAR and regression, the significance being stated at $\mathrm{p}<0.05$. Results: Trimester wise evolution of hematologic status in anemic women shows a rise in values of hemoglobin (+average $1 \mathrm{gr} / \mathrm{dl}$ ) and hematocrit $(+3 \%)$ throughout pregnancy, with significant change only between recruitment and 28 - 32 weeks. In non anemic women a significant decrease was observed, although levels remained normal. For ferritin (7.5 - 53 $\mathrm{ng} / \mathrm{mL}$ from recruitment to term) and transferrin (107 - $157 \mathrm{~g} / \mathrm{L})$, significant rise was found in anemic women from recruitment to $28-32$ weeks, while in
\end{abstract}


non anemic notable change occurred only for transferrin (133 - $204 \mathrm{~g} / \mathrm{L})$. Serum iron significantly increased $(53-83 \mu \mathrm{g} / \mathrm{dL})$ from recruitment to $28-32$ weeks in anemic women only. So, following supplementation, values of blood parameters tended to be similar for both goups at term. Non anemic women did not benefit from supplementation. SOD decreased and LDL values increased alongside supplementation. This represents a growing oxidant threat for both anemic and non anemic women. This could be related to iron overload and/or unabsorbed iron. Conclusion: Iron supplementation improved iron status in anemic women, not in non anemic. Differences that were significant between groups at recruitment disappeared by 28 - 32 weeks. Non anemic women did not benefit from supplementation. SOD decreased and LDL values increased alongside supplementation. This represents a growing oxidant threat for both anemic and non anemic women. This could be related to iron overload and/or unabsorbed iron. Doses of iron should be decreased (at least halved) and duration of treatment restricted to not more than 12 weeks.

\section{Keywords}

Iron Supplementation, Pregnancy, Iron And Oxidative Status, Kinshasa

\section{Introduction}

Routine iron supplementation during pregnancy is a worldwide practice among strategies to prevent the burden of maternal anemia. Although some studies pointed out the risk of iron overload likely to enhance the Fenton reaction [1], supplementation used to be provided without prior assessment of iron and oxidative status of women concerned.

In a previous study [2] we assessed baseline iron and oxidative status of pregnant women just before the period scheduled to start routine iron supplementation in our setting. We found that the majority of pregnant women were anemic. Values observed for markers of iron status (hemoglobin, serum iron and ferritin) were significantly lower, while those of oxidative status (superoxide dismutase or SOD and oxidized LDL) were significantly higher in anemic women. This confirms the link between maternal anemia and oxidative stress.

The rate and amplitude of this situation are expected to rise over pregnancy, due to maternal and fetal physiologic demand. Indeed, ferritin that reflects total iron in the body has been found decreasing over normal pregnancy [1] and hepcidin, the master protein regulating the iron bioavailability, has also been found declining [3]. The lower the maternal serum hepcidin, the higher the rate of mother-to-infant iron transfer [4]. Consequently, most of studies that monitored the evolution of hematologic variables during normal pregnancy found significant decrease of mean hemoglobin, serum iron and ferritin values from first to third trimesters [5]. So, it can be assumed that the oxidative status also varies accordingly, although similar studies assessing oxidative status throughout 
pregnancy are rare.

For routine supplementation, the quantity of iron to be given individually varies a lot from country to country: this can fluctuate from $30 \mathrm{mg} /$ day [6] to 160 $200 \mathrm{mg} /$ day, although $100 \mathrm{mg} /$ day has been claimed to increase oxidative stress in India [7]. Resulting improvement in both iron and oxidative status is not as yet obvious in the literature that reports conflicting findings [8] [9] [10]. In Africa, there is no study dealing with iron and oxidative status changes alongside routine iron administration. In Egypt, Aly et al. [11], prescribed iron only for curative purpose in anemic patients $(100 \mathrm{mg}$ or $200 \mathrm{mg}$ of ferrous sulfate twice a day) and women were not assessed at the same gestational ages between 20th and 36 th gestational weeks. After 8 weeks of supplementation they observed a significant increase in hemoglobin, serum ferritin as well as in pro-oxidants and total antioxidant capacity. So far, no other African study related to this issue can be found.

Addressing routine (prophylactic and curative) supplementation during pregnancy, the present study aimed to assess variations of indices of iron and oxidative status, while taking in account some factors such as nutritional intake and intestinal conditions of iron absorption.

\section{Methods}

The current study was approved by the Ethics Committee of the School of Public Health of the University of Kinshasa, DR Congo, and was conducted during 10 months (from September 2017 throughout June 2018), the protocol of which was recently published [12]. The study sample was represented by pregnant women with a singleton pregnancy not exceeding 19 weeks (starting point of iron supplementation), without obvious pathology likely to have a negative influence on pregnancy (hypertension, diabetes mellitus, sickle cell disease, HIV, malaria, respiratory insufficiency) and regardless of age and parity. Eligible women were definitely recruited after informed and written consent to participate in the study. The sampling was carried out consecutively for all pregnant women presenting themselves at prenatal care until completion of 74 women (the minimum size of 54 as calculated according to appropriate formulas +20 women for potential drop out). It is a prospective observational one concerning a cohort of 74 healthy pregnant women with singleton pregnancy of $<19$ weeks of gestation whose baseline iron and oxidative status have been recently assessed at university clinics of Kinshasa, DR Congo [2]. Only women reaching fetal viability (28 weeks of gestation) were selected. Irrespective of their biochemical status, all women of this cohort received routine Fe supplementation according to the national policy. Women with anemia $(\mathrm{Hb}<10 \mathrm{~g} \%)$ took iron tablets for curative purposes (320 mg/day of ferric ammonium citrate), while the others received preventive iron supplementation (160 mg/day of ferric ammonium citrate). Let us note that these dosages have never been questioned in the country. Iron was associated with $15 \mathrm{mg}$ folate/day. 
Nutritional iron intake was measured through a 24-hour recall using a questionnaire of common foods, including stuffs and habits likely to impede iron absorption, such as pica/geophagia (proven to contain kaolinite, calcic phosphates, quartz, polyphenols with chelating properties [13], tannins and fibers [14]. Supplemental iron intake was determined with a sheet containing details on the duration of the treatment. A direct microscopic examination of stool was performed at recruitment.

Additionally to baseline measurements at recruitment ( \pm 15 weeks of gestation) blood sampling and analysis were carried out at 28 - 32 weeks and at term in conditions previously described [2]. Biological parameters were: hemoglobin, hematocrit, ferritin, serum iron, transferrin and iron saturation capacity for iron status and SOD, uric acid, oxidized LDL and fasting blood glucose for oxidative status.

Data were registered using Microsoft Excel (Microsoft Corporation, Redmond, WA, USA, 2007) and after transfer to SPSS (version 18.0; SPSS Inc., Chicago, IL, USA). For statistical calculations we used t-test and chi-square test to compare means and proportions, respectively, ANOVAR to assess differences of means between periods of pregnancy and odds ratio to check associations between anemia/oxidative stress and factors related to iron absorption. Univariate analysis (odds ratios [ORs]) were stratified in a dichotomous way (with or without anemia, with and without oxidative stress, with and without iron/oxidative status variations) to seek significant association with iron supplements. Multivariate calculations (regression) used various durations of supplementation to seek the lowest one at which an OR $\geq 1.5$ with $95 \%$ confidence intervals (CIs) and p-value $<0.05$ could be found for oxidative status.

\section{Results}

The mean age of the study group $(\mathrm{n}=74)$ was $32.2 \pm 5.2$ years, with 39 women diagnosed anemic $(52.2 \%)$ according to local standards (hemoglobin $<10 \mathrm{~g} / \mathrm{dl}$ ). The majority was para 2, with average weight of $70.2 \pm 14.5 \mathrm{Kg}$ (P not significant between sub-groups) and BMI of $26 \pm 5.2 \mathrm{Kg} / \mathrm{m}^{2}(\mathrm{p}<0.01)$. The proportion of obeses was $18.9 \%$. Of their general characteristics already presented in a previous paper [2], Table 1 includes those for which significant differences were observed at recruitment between anemic and non anemic women, namely socioeconomic status (lower in anemic group), BMI (higher in anemic group), nutritional iron intake (lower in anemic group) and intestinal parasitosis (more frequent in anemic group). The duration of supplementation and eating habits likely to impede iron absorption (Geophagia, fibers and tannins consumption) were also included.

Additional significant differences between anemic and non anemic women were found in consumption of tannins and geophagia (more frequent in anemic women). As pregnancy progressed pairwise comparison based on the anemic state varied trimester wise (Table 1). It appeared that differences based on the anemic state remained significant throughout pregnancy for high consumption 
Table 1. General characteristics of the study group throughout pregnancy.

\begin{tabular}{|c|c|c|c|c|c|c|c|}
\hline Characteristics & & & & & & & \\
\hline Sociodemographic & & $\mathrm{n}$ & $14-19$ GA & $\mathrm{n}$ & $28-32 \mathrm{GA}$ & $\mathrm{n}$ & At term \\
\hline \multirow{4}{*}{ Low socioeconomic level } & Anemic & 39 & $69.2 \% y$ & 44 & $54.5 \%$ & 39 & $46.2 \%$ \\
\hline & Non anemic & 35 & $2.9 \%$ & 30 & $13.3 \%$ & 35 & $28.6 \%$ \\
\hline & Overall group & 74 & $37.8 \%$ & 74 & $37.8 \%$ & 74 & $37.8 \%$ \\
\hline & $\mathrm{p}$ & & $<0.001$ & & $<0.001$ & & 0.094 \\
\hline \multirow{5}{*}{$\begin{array}{l}\text { Iron nutritional intake } \\
\text { (gr) }\end{array}$} & Anemic & 39 & $22.8 \pm 4.9$ & 44 & $36.8 \pm 11.7$ & 39 & $41.4 \pm 11.1$ \\
\hline & Non anemic & 35 & $31.4 \pm 9.5$ & 30 & $39.8 \pm 10.7$ & 35 & $40.9 \pm 11.9$ \\
\hline & Overall group & 74 & $26.9 \pm 8.6$ & 74 & $38.02 \pm 11.4$ & 74 & $41.2 \pm 11.4$ \\
\hline & $\mathrm{p}$ & & $<0.001$ & & 0.262 & & 0.871 \\
\hline & Anemic & 39 & $76.9 \%$ & 44 & $50 \%$ & 39 & $59 \%$ \\
\hline \multirow[t]{4}{*}{ Fibers and tannins consumption } & Non anemic & 35 & $25.7 \%$ & 30 & $16.7 \%$ & 35 & $17.1 \%$ \\
\hline & Overall group & 74 & $52.7 \%$ & 74 & $36.5 \%$ & 74 & $39.2 \%$ \\
\hline & $\mathrm{p}$ & & $<0.001$ & & $<0.004$ & & $<0.001$ \\
\hline & Anemic & 39 & $41 \%$ & 44 & $40.9 \%$ & 39 & $35.9 \%$ \\
\hline \multirow[t]{3}{*}{ Geophagia } & Non anemic & 35 & $8.6 \%$ & 30 & $16.7 \%$ & 35 & $11.4 \%$ \\
\hline & Overall group & 74 & $25.7 \%$ & 74 & $31.1 \%$ & 74 & $24.3 \%$ \\
\hline & $\mathrm{p}$ & & $<0.002$ & & $<0.03$ & & $<0.02$ \\
\hline \multicolumn{8}{|l|}{ Clinical } \\
\hline \multirow{4}{*}{$\begin{array}{c}\text { BMI } \\
\left(\mathrm{Kg} / \mathrm{m}^{2}\right)\end{array}$} & Anemic & 39 & $27.8 \pm 5.4$ & 44 & $27.3 \pm 5.7$ & 39 & $26.9 \pm 5.7$ \\
\hline & Non anemic & 35 & $24.03 \pm 4.3$ & 30 & $24.1 \pm 3.7$ & 35 & $25 \pm 4.5$ \\
\hline & Overall group & 74 & $26 \pm 5.2$ & 74 & $26 \pm 5.2$ & 74 & $26 \pm 5.2$ \\
\hline & $\mathrm{p}$ & & $<0.003$ & & $<0.01$ & & 0.117 \\
\hline \multirow{5}{*}{$\begin{array}{l}\text { Duration of supplementation } \\
\text { (weeks) }\end{array}$} & Anemic & 39 & - & 44 & $12.3 \pm 2.5$ & 39 & $8.6 \pm 2.8$ \\
\hline & Non anemic & 35 & - & 30 & $10.6 \pm 1.8$ & 35 & $8.6 \pm 2.3$ \\
\hline & Overall group & 74 & - & 74 & $11.6 \pm 2.4$ & 74 & $8.6 \pm 2.5$ \\
\hline & $\mathrm{p}$ & & - & & $<0.003$ & & 0.976 \\
\hline & Anemic & 39 & $61.5 \%$ & 44 & $79.5 \%$ & 39 & $64.1 \%$ \\
\hline \multirow[t]{3}{*}{ Intestinal parasitosis } & Non anemic & 35 & $34.3 \%$ & 30 & $30 \%$ & 35 & $8.6 \%$ \\
\hline & Overall group & 74 & $48.6 \%$ & 74 & $59.5 \%$ & 74 & $37.8 \%$ \\
\hline & $\mathrm{p}$ & & $<0.02$ & & $<0.001$ & & $<0.001$ \\
\hline
\end{tabular}

of tannins and geophagia and intestinal parasitosis, while only till 28 - 32 weeks for low socioeconomic level and just at recruitment (14 - 19 weeks) for low nutritional iron intake.

General characteristics proven to impede intestinal iron absorption such ac pica, fibers and tannins were found significantly more consumed in the anemic group. The duration of iron replacement was significantly longer in the anemic 
group by 28 - 32 weeks $(\mathrm{p}<0.003)$ owed to the fact that curative supplementation was started at 19 weeks of gestation while preventive supply was initiated at recruitment (15.8 weeks of gestation).

Trimester wise evolution of hematologic status as presented in Table 2 shows a rise in values of hemoglobin (+average $1 \mathrm{gr} / \mathrm{dl}$ ) and hematocrit $(+3 \%)$ in anemic women, with significant change only between recruitment and 28 - 32 weeks. So, the proportion of anemic women decreased following supplementation. In non anemic a significant decrease was observed from recruitment to 28 32 weeks, although levels remained normal. They rose again thereafter till term.

For ferritin (7.5 - $53 \mathrm{ng} / \mathrm{mL}$ from recruitment to term) and transferrin (107 $157 \mathrm{~g} / \mathrm{L}$ ) significant rise was found in anemic women from recruitment to 28 32 weeks, while in non anemic a small decline was noted at mid-pregnancy before rising again till term. Notable change (rise) occurred for transferrin throughout pregnancy for both groups. Serum iron significantly increased (53$83 \mu \mathrm{g} / \mathrm{dl}$ ) from recruitment to 28 - 32 weeks in anemic women only. So, differences that were significant between groups at recruitment disappeared thereafter, and blood parameters tended to be similar for both groups at term. Non anemic women did not benefit from supplementation.

As shown in Table 3 that presents evolution of oxidative status alongside iron supplementation, SOD values decreased alongside supplementation, although no significant difference was observed trimester wise. At term, values were still higher in anemic but difference was no more significant in comparison to non anemic women.

For oxidized LDL, both in anemic and non anemic women, values significantly increased from recruitment to term. Values remained significantly higher in anemic women.

An increase was also observed in uric acid from recruitment to mid-pregnancy among anemic women and in glycemia in non anemic.

Furthering the role of iron supplementation on changes of iron and oxidative status, we also questioned changes (at 28 - 32 weeks of gestation and at term) in factors for which significant differences have been found between anemic and non anemic groups (Table 4, Table 5). At 28 - 32 weeks of gestation the low socioeconomic status ( $\mathrm{OR}=7.8$; CI 95\%: $2.3-26.1 ; \mathrm{p}<0.001$ ) and consumption of tannins and fibers $(\mathrm{OR}=3.9$; CI 95\%: $1.5-10.3 ; \mathrm{p}<0.01)$ were significantly associated with anemia. At term only the consumption of tannins and fibers remained associated (OR 2.7; CI 95\%: 1.1 - 6.9; $\mathrm{p}<0.04$ ).

For oxidative stress (Table 5), we observed no significant associations after recruitment for SOD, while, for oxidized LDL all factors but geophagia remained associated with oxidative stress till term.

The association of oxidative stress (SOD $<785$ or $>1570$ UI/L; oxidized LDL $<$ 200 or $>600 \mathrm{UI} / \mathrm{L}$ ) with the duration of supplementation is presented in Table 6 . The duration extended from 12 to 27 weeks, with mean of $20.2 \pm 2.6$ weeks. Calculations (regression) were made week wise under 27 weeks in order to find the lowest duration at which an $\mathrm{OR} \geq 1.5$ with $\mathrm{p}$-value $<0.05$ could be found. 
Table 2. Variations of iron status following iron supplementation.

\begin{tabular}{|c|c|c|c|c|c|c|c|c|c|}
\hline Markers & & $\mathrm{n}$ & $14-19 \mathrm{GA}^{(1)}$ & $28-32 \mathrm{SA}^{(2)}$ & At term ${ }^{(3)}$ & $\mathrm{p}^{(1-2)}$ & $\mathrm{p}^{(1-3)}$ & $\mathrm{p}^{2-3)}$ & $\mathrm{p}^{(1-2-3)}$ \\
\hline & Anemic & 39 & $9.01 \pm 0.6$ & $9.6 \pm 0.6$ & $9.7 \pm 0.8$ & $<0.001$ & $<0.001$ & 0.498 & $<0.001$ \\
\hline \multirow[t]{4}{*}{ Hemoglobin (mg/dl) } & Non anemic & 35 & $11.6 \pm 0.9$ & $10.2 \pm 0.8$ & $10.3 \pm 0.8$ & $<0.001$ & $<0.001$ & 0.652 & $<0.001$ \\
\hline & Overall group & 74 & $10.3 \pm 1.5$ & $9.9 \pm 0.8$ & $9.9 \pm 0.9$ & 0.066 & 0.191 & 0.463 & 0.115 \\
\hline & $\mathrm{p}$ & & $<0.001$ & $<0.001$ & 0.001 & & & & \\
\hline & Anemic & 39 & $26.3 \pm 2.2$ & $29.2 \pm 2.02$ & $29.4 \pm 2.9$ & $<0.001$ & $<0.001$ & 0.715 & $<0.001$ \\
\hline \multirow[t]{4}{*}{ Hematocrit (\%) } & Non anemic & 35 & $35.1 \pm 2.7$ & $30.5 \pm 2.9$ & $31 \pm 2.6$ & $<0.001$ & $<0.001$ & 0.411 & $<0.001$ \\
\hline & Overall group & 74 & $30.5 \pm 5.04$ & $29.8 \pm 2.5$ & $30.2 \pm 2.8$ & 0.312 & 0.656 & 0.411 & 0.540 \\
\hline & $\mathrm{p}$ & & $<0.001$ & $<0.03$ & $<0.02$ & & & & \\
\hline & Anemic & 39 & $7.5 \pm 3.9$ & $22.5 \pm 29.2$ & $52.9 \pm 106.8$ & $<0.003$ & $<0.02$ & 0.091 & $<0.01$ \\
\hline \multirow[t]{4}{*}{ Ferritin (ng/mL) } & Non anemic & 35 & $35.7 \pm 17.1$ & $25.3 \pm 30.4$ & $41.8 \pm 71.2$ & 0.080 & 0.625 & 0.210 & 0.314 \\
\hline & Overall group & 74 & $20.9 \pm 18.6$ & $23.8 \pm 29.6$ & $47.6 \pm 91.1$ & 0.469 & $<0.02$ & $<0.04$ & $<0.01$ \\
\hline & $\mathrm{p}$ & & $<0.001$ & 0.697 & 0.606 & & & & \\
\hline & Anemic & 39 & $52.7 \pm 38.9$ & $83.4 \pm 49.7$ & $81.2 \pm 50.2$ & $<0.01$ & $<0.01$ & 0.850 & $<0.01$ \\
\hline \multirow[t]{4}{*}{ Serum iron $(\mu \mathrm{g} / \mathrm{dl})$} & Non anemic & 35 & $96.2 \pm 41.9$ & $80.1 \pm 41.4$ & $80.5 \pm 37.4$ & 0.109 & 0.101 & 0.967 & 0.165 \\
\hline & Overall group & 74 & $73.3 \pm 45.7$ & $81.8 \pm 45.7$ & $80.9 \pm 44.3$ & 0.257 & 0.306 & 0.898 & 0.454 \\
\hline & $\mathrm{p}$ & & $<0.001$ & 0.759 & 0.942 & & & & \\
\hline & Anemic & 39 & $107.2 \pm 45.9$ & $166.7 \pm 94.4$ & $157.4 \pm 89.9$ & $<0.01$ & $<0.01$ & 0.657 & $<0.01$ \\
\hline \multirow[t]{4}{*}{ Transferrin $(\mathrm{g} / \mathrm{L})$} & Non anemic & 35 & $133.1 \pm 91.9$ & $178.3 \pm 119.5$ & $203.8 \pm 120.4$ & 0.081 & $<0.01$ & 0.377 & $<0.04$ \\
\hline & Overall group & 74 & $119.5 \pm 72.2$ & $172.2 \pm 106.4$ & $179.3 \pm 107.3$ & $<0.01$ & $<0.001$ & 0.685 & $<0.001$ \\
\hline & $\mathrm{p}$ & & 0.125 & 0.644 & 0.063 & & & & \\
\hline & Anemic & 39 & $2.5 \pm 1.1$ & $2.4 \pm 1.4$ & $2.3 \pm 1.3$ & 0.671 & 0.421 & 0.747 & 0.746 \\
\hline \multirow[t]{3}{*}{ CST (\%) } & Non anemic & 35 & $2.8 \pm 1.1$ & $2.3 \pm 1.1$ & $2.1 \pm 1.2$ & 0.075 & $<0.02$ & 0.450 & $<0.04$ \\
\hline & Overall group & 74 & $2.6 \pm 1.1$ & $2.3 \pm 1.3$ & $2.2 \pm 1.3$ & 0.136 & $<0.03$ & 0.466 & 0.077 \\
\hline & $\mathrm{p}$ & & 0.357 & 0.678 & 0.422 & & & & \\
\hline
\end{tabular}

It can be seen that significant associations with oxidative stress appeared only for oxidized LDL after $\geq 20$ weeks of supplementation (OR = 7.04; CI95\%: 2.5 19.7; $\mathrm{p}<0.001)$. This concerns mostly anemic women for which iron was provided earlier than non anemic.

Table 7 presents associations of anemia with nutritional iron intake. It was found a significant association under $47 \mathrm{mg} / \mathrm{day}(\mathrm{OR}=2.3$; IC 95\%: $1.7-2.9 ; \mathrm{p}<$ $0.05)$ at recruitment, under $35 \mathrm{mg} /$ day $(\mathrm{OR}=4.9$; IC 95\%: $1.2-20.7 ; \mathrm{p}<0.03)$ at 28 - 32 weeks and not thereafter.

Table 8 shows the associations of nutritional iron intake with oxidative stress (pathologic values of SOD and oxidized LDL considered).

At recruitment, significant association was found under $26 \mathrm{mg} /$ day for SOD $(\mathrm{OR}=1.7 ; \mathrm{CI} 95 \%: 1.2-2.5 ; \mathrm{p}<0.001)$ and oxidized LDL (OR = 1.8; CI 95\%: 1.01 - 3.2; $\mathrm{p}<0.04)$. Considering oxidative status at $28-32$ weeks of gestation and at 
Table 3. Variations of oxidative status following iron supplementation.

\begin{tabular}{|c|c|c|c|c|c|c|c|c|c|}
\hline Markers & & $\mathrm{n}$ & $14-19 \mathrm{GA}^{(1)}$ & $28-32 \mathrm{GA}^{(2)}$ & At term ${ }^{(3)}$ & $\mathrm{p}^{(1-2)}$ & $\mathrm{p}^{(1-3)}$ & $\mathrm{p}^{(2-3)}$ & $\mathrm{p}^{(1-2-3)}$ \\
\hline \multirow{4}{*}{ SOD (UI/L) } & Anemic & 39 & $1056.4 \pm 762.1$ & $917.3 \pm 629.1$ & $808.8 \pm 560.3$ & 0.382 & 0.106 & 0.424 & 0.252 \\
\hline & Non anemic & 35 & $682.6 \pm 543.9$ & $635.4 \pm 483.2$ & $777.4 \pm 508.7$ & 0.702 & 0.454 & 0.235 & 0.501 \\
\hline & Overall group & 74 & $879.6 \pm 689.5$ & $783.9 \pm 578.7$ & $793.9 \pm 533.1$ & 0.362 & 0.399 & 0.913 & 0.572 \\
\hline & $\mathrm{p}$ & & $<0.02$ & $<0.04$ & 0.802 & & & & \\
\hline \multirow{4}{*}{$\begin{array}{l}\text { Oxidized LDL } \\
\text { (UI/L) }\end{array}$} & Anemic & 39 & $439.6 \pm 209.5$ & $512.1 \pm 253.7$ & $684.1 \pm 222.1$ & 0.147 & $<0.001$ & $<0.01$ & $<0.001$ \\
\hline & Non anemic & 35 & $192.8 \pm 136.3$ & $352.1 \pm 214.6$ & $360.2 \pm 215.4$ & $<0.01$ & $<0.001$ & 0.852 & $<0.01$ \\
\hline & Overall group & 74 & $322.9 \pm 216.5$ & $436.4 \pm 247.8$ & $530.9 \pm 271.7$ & $<0.01$ & $<0.001$ & $<0.03$ & $<0.001$ \\
\hline & $\mathrm{p}$ & & $<0.001$ & $<0.01$ & $<0.001$ & & & & \\
\hline \multirow{4}{*}{$\begin{array}{l}\text { Uric acid } \\
(\mathrm{mg} / \mathrm{L})\end{array}$} & Anemic & 39 & $2.6 \pm 1.03$ & $3.6 \pm 1.7$ & $1.9 \pm 1.3$ & $<0.01$ & $<0.001$ & 0.248 & $<0.01$ \\
\hline & Non anemic & 35 & $3.03 \pm 0.9$ & $5.3 \pm 11.01$ & $6.02 \pm 2.4$ & 0.078 & 0.005 & 0.361 & 0.028 \\
\hline & Overall group & 74 & $2.8 \pm 1.01$ & $4.4 \pm 7.7$ & $4.4 \pm 1.3$ & $<0.01$ & $<0.001$ & 0.138 & $<0.01$ \\
\hline & $\mathrm{p}$ & & 0.088 & 0.326 & 0.356 & & & & \\
\hline \multirow{4}{*}{$\begin{array}{c}\text { Fasting } \\
\text { glycemia } \\
(\mathrm{g} / \mathrm{L})\end{array}$} & Anemic & 39 & $75.3 \pm 15.9$ & $80.3 \pm 21.9$ & $81.4 \pm 17.9$ & 0.256 & 0.115 & 0.799 & 0.313 \\
\hline & Non anemic & 35 & $73.4 \pm 15.9$ & $82.6 \pm 18,4$ & $82 \pm 20.4$ & $<0.04$ & 0.055 & 0.903 & 0.071 \\
\hline & Overall group & 74 & $74.4 \pm 15.8$ & $81.4 \pm 20.2$ & $81.7 \pm 18.9$ & $<0.03$ & $<0.02$ & 0.917 & $<0.03$ \\
\hline & $\mathrm{p}$ & & 0.614 & 0.626 & 0.895 & & & & \\
\hline
\end{tabular}

Table 4. Changes in factors associated with anemia throughout pregnancy.

\begin{tabular}{|c|c|c|c|c|c|c|c|c|c|c|c|c|}
\hline \multirow{3}{*}{ Factors } & \multicolumn{12}{|c|}{ Anemia } \\
\hline & \multicolumn{4}{|c|}{14 - 19 gestational weeks } & \multicolumn{4}{|c|}{28 - 32 gestational weeks } & \multicolumn{4}{|c|}{ At term } \\
\hline & $\begin{array}{l}\text { Frequency } \\
(\mathrm{n}=39)\end{array}$ & OR & CI $(95 \%)$ & p & $\begin{array}{l}\text { Frequency } \\
(\mathrm{n}=44)\end{array}$ & OR & CI $(95 \%)$ & $\mathrm{p}$ & $\begin{array}{l}\text { Frequency } \\
(\mathrm{n}=39)\end{array}$ & OR & CI (95\%) & $\mathrm{p}$ \\
\hline $\begin{array}{c}\text { Low socioeconomic } \\
\text { level }\end{array}$ & $27(69.2 \%)$ & 20.7 & $2.9-142.9$ & $<0.001$ & $24(54.5 \%)$ & 7.8 & $2.3-26.1$ & $<0.001$ & $18(46.2 \%)$ & 2.1 & $0.8-5.6$ & 0.094 \\
\hline Intestinal parasitosis & $24(61.5 \%)$ & 3.1 & $1.2-7.9$ & $<0.02$ & $22(50 \%)$ & 1.1 & $0.5-2.9$ & 0.482 & $18(46.2 \%)$ & 0.8 & $0.3-2.02$ & 0.413 \\
\hline Geophagia & $16(41 \%)$ & 7.4 & $1.9-28.5$ & $<0.01$ & $14(31.8 \%)$ & 2.3 & $0.7-7.4$ & 0.115 & $13(33.3 \%)$ & 2.4 & $0.8-7.3$ & 0.092 \\
\hline Tanins and fibers & $30(76.9 \%)$ & 9.6 & $3.3-27.9$ & $<0.001$ & $29(65.9 \%)$ & 3.9 & $1.5-10.3$ & $<0.01$ & 25 (64.1\%) & 2.7 & $1.1-6.9$ & $<0.04$ \\
\hline
\end{tabular}

term the association appeared significant for oxidized LDL at $19 \mathrm{mg} /$ day $(\mathrm{OR}=2.2$; CI 95\%: $1.7-2.8 ; \mathrm{p}<0.01$ and $\mathrm{OR}=1.9 ; \mathrm{CI} 95 \%: 1.5-2.4 ; \mathrm{p}<0.02$, respectively).

\section{Discussion}

Our study aimed to assess variations of iron and oxidative status of routinely supplemented women during pregnancy. In anemic women of our series, evolution of hematologic parameters showed an increase in hemoglobin and hematocrit values (+average $1 \mathrm{gr} / \mathrm{dl}$ and $+3 \%$, respectively) throughout pregnancy, with significant change only between recruitment and 28 - 32 weeks. Nutrition was an important factor in the generation of anemia found in our series, as it can be derived from significant associations of anemia with nutritional iron intake (under $47 \mathrm{mg} /$ day at recruitment, under $35 \mathrm{mg} /$ day at $28-32$ weeks and not thereafter). 
Table 5. Changes in factors associated with oxidative stress throughout pregnancy.

\begin{tabular}{|c|c|c|c|c|c|c|c|c|c|}
\hline \multirow{2}{*}{ Factors } & \multicolumn{3}{|c|}{$14-19$ weeks } & \multicolumn{3}{|c|}{$28-32$ weeks } & \multicolumn{3}{|c|}{ At term } \\
\hline & Frequency & OR (CI 95\%) & $\mathrm{p}$ & Frequency & OR (CI 95\%) & $\mathrm{p}$ & Frequency & OR (CI 95\%) & $\mathrm{p}$ \\
\hline Anemia & $38 / 60(63.3 \%)$ & $22.5(2.8-183.5)$ & $<0.001$ & $26 / 52(50 \%)$ & $0.7(0.3-1.9)$ & 0.323 & $26 / 48(54.2 \%)$ & $1.2(0.5-3.1)$ & 0.460 \\
\hline Low socioeconomic level & $27 / 60(45 \%)$ & $10.6(1.3-86.6)$ & $<0.01$ & $20 / 52(38.5 \%)$ & $1.1(0.4-3.1)$ & 0.540 & $20 / 48(41.7 \%)$ & $1.6(0.6-4.4)$ & 0.252 \\
\hline Intestinal parasitosis & $32 / 60(53.3 \%)$ & $2.9(0.8-10.1)$ & 0.084 & $23 / 52(44.2 \%)$ & $0.6(0.2-1.5)$ & 0.180 & $23 / 48(47.9 \%)$ & $0.9(0.4-2.4)$ & 0.529 \\
\hline Geophagia & $18 / 60(30 \%)$ & $5.6(0.7-45.8)$ & 0.070 & $14 / 52(26.9 \%)$ & $1.3(0.4-4.04)$ & 0.473 & $14 / 48(29.2 \%)$ & $1,7(0.5-5.5)$ & 0.259 \\
\hline \multirow[t]{2}{*}{ Tannins and fibers } & $35 / 60(58.3 \%)$ & $3.5(1.9-12.4)$ & $<0.05$ & $30 / 52(57.7 \%)$ & $1.9(0.7-5.4)$ & 0.143 & $26 / 48(54.2 \%)$ & $1.2(0.5-3.1)$ & 0.460 \\
\hline & \multicolumn{9}{|c|}{ Patholologic oxidized LDL } \\
\hline Low socioeconomic level & $25 / 41(61 \%)$ & $\begin{array}{r}15.6(4.1- \\
59.8)\end{array}$ & $<0.001$ & $21 / 38(55.3)$ & $5.1(1.8-14.5)$ & $<0.01$ & $25 / 42(59.5 \%)$ & $14.2(3.7-54.2)$ & $<0.001$ \\
\hline Intestinal parasitosis & $25 / 41(61 \%)$ & $3.1(1.2-8.1)$ & $<0.02$ & $22 / 38(57.9 \%)$ & $2.2(0.9-5.5)$ & 0.080 & $26 / 42(61.9 \%)$ & $3.6(14-9.5)$ & $<0.01$ \\
\hline Geophagia & $14 / 41(34.1 \%)$ & $2.9(1.9-9.2)$ & $<0.05$ & $12 / 38(31.6 \%)$ & $1.9(0.7-5.6)$ & 0.177 & $15 / 42(35.7 \%)$ & $3.9(1.2-13.2)$ & $<0.03$ \\
\hline Tannins and fibers & $27 / 41(65.9 \%)$ & $3.4(1.3-8.8)$ & $<0.02$ & $26 / 38(68.4 \%)$ & $3.8(1.5-10.1)$ & $<0.01$ & $30 / 42(71.4 \%)$ & $6.4(2.3-17.7)$ & $<0.001$ \\
\hline
\end{tabular}

Table 6. Association of duration of iron supplementation with oxidative stress.

\begin{tabular}{|c|c|c|c|}
\hline Duration of supplementation (weeks) & Frequency & OR (CI 95\%) & $\mathrm{p}$ \\
\hline & \multicolumn{3}{|c|}{ Pathologic SOD } \\
\hline$\geq 24$ & $3 / 48(6.3 \%)$ & $2.1(0.8-5.3)$ & $<0.05$ \\
\hline$\geq 23$ & $7 / 48(14.6 \%)$ & $1.4(0.8-2.4)$ & 0.163 \\
\hline$\geq 22$ & $11 / 48(22.9 \%)$ & $1,2(0.8-1.8)$ & 0.319 \\
\hline$\geq 21$ & $18 / 48(37.5 \%)$ & $1,1(0.8-1.6)$ & 0.316 \\
\hline$\geq 20$ & $25 / 48(52.1 \%)$ & $1,2(0.9-1.7)$ & 0.196 \\
\hline \multirow[t]{2}{*}{$\geq 19$} & $37 / 48(77.1 \%)$ & $1.2(0.8-1.7)$ & 0.326 \\
\hline & \multicolumn{3}{|c|}{ Pathologic oxidized LDL } \\
\hline$\geq 24$ & $8 / 42(19 \%)$ & $7.3(0.9-61.7)$ & $<0.04$ \\
\hline$\geq 23$ & $13 / 42(31 \%)$ & $13.9(1.7-113.03)$ & $<0.01$ \\
\hline$\geq 22$ & $18 / 42(42.9 \%)$ & $23.3(2.9-186.7)$ & $<0.01$ \\
\hline$\geq 21$ & $24 / 42(57.1 \%)$ & $5.8(1.9-16.9)$ & $<0.01$ \\
\hline$\geq 20$ & $32 / 42(76.2 \%)$ & $7.04(2.5-19.7)$ & $<0.001$ \\
\hline$\geq 19$ & $34 / 42(81 \%)$ & $1.2(0.4-3.7)$ & 0.494 \\
\hline
\end{tabular}

General characteristics proven to impede intestinal iron absorption such as pica, fibers and tannins significantly more frequent in the anemic group could be accounted for among factors influencing the responsiveness of supplemented women. The effect of intestinal parasitosis was probably offset by treatment at recruitment. Finally, the proportion of anemic women decreased following 
Table 7. Associations of anemia with nutritional iron intake.

\begin{tabular}{|c|c|c|c|c|c|}
\hline $\begin{array}{c}\text { Nutritonal iron intake } \\
(\mathrm{mg} / \text { day })\end{array}$ & $\begin{array}{l}\text { Period (weeks } \\
\text { of gestation) }\end{array}$ & $\begin{array}{c}\text { Frequency of } \\
\text { anemia }\end{array}$ & OR & CI $95 \%$ & $\mathrm{p}$ \\
\hline \multirow{3}{*}{$<48$} & $14-19$ & $39 / 39(100 \%)$ & 2.2 & $1.7-2.9$ & 0.101 \\
\hline & $28-32$ & $43 / 44(97.7 \%)$ & 3.1 & $0.3-35.5$ & 0.358 \\
\hline & At term & $38 / 39(97.4 \%)$ & 2.3 & $0.2-26.6$ & 0.459 \\
\hline \multirow{3}{*}{$<47$} & $14-19$ & $39 / 39$ (100\%) & 2.3 & $1.7-2.9$ & $<0.05$ \\
\hline & $28-32$ & $43 / 44(97.7 \%)$ & 4.8 & $0.5-48.3$ & 0.179 \\
\hline & A term & $38 / 39(97.4 \%)$ & 3.6 & $0.4-35.9$ & 0.267 \\
\hline \multirow{3}{*}{$<35$} & $14-19$ & $38 / 39(97.4 \%)$ & 15.2 & $1.8-126.2$ & $<0.01$ \\
\hline & $28-32$ & $41 / 44(93.2 \%)$ & 4.9 & $1.2-20.7$ & $<0.03$ \\
\hline & A term & $35 / 39(89.7 \%)$ & 2.2 & $0.6-8.2$ & 0.198 \\
\hline \multirow{3}{*}{$<21$} & $14-19$ & $11 / 39(28.2 \%)$ & 13.4 & $1.6-109.9$ & $<0.01$ \\
\hline & $28-32$ & $11 / 44(25 \%)$ & 9.7 & $1.2-79.5$ & $<0.02$ \\
\hline & At term & $8 / 39(20.5 \%)$ & 2 & $0.6-7.3$ & 0.230 \\
\hline \multirow{3}{*}{$<19$} & $14-19$ & $7 / 39(17.9 \%)$ & 0.5 & $0.4-0.6$ & $<0.01$ \\
\hline & $28-32$ & $7 / 44(15.9 \%)$ & 0.6 & $0.5-0.7$ & $<0.03$ \\
\hline & At term & $5 / 39(12.8 \%)$ & 2.4 & $0.4-13.4$ & 0.262 \\
\hline \multirow{3}{*}{$<18$} & $14-19$ & $4 / 39(10.3 \%)$ & 0.5 & $0.4-0.6$ & 0.071 \\
\hline & $28-32$ & $4 / 44(9.1 \%)$ & 0.6 & $0.5-0.7$ & 0.118 \\
\hline & At term & $3 / 39(7.7 \%)$ & 2.8 & $0.3-28.6$ & 0.349 \\
\hline
\end{tabular}

supplementation. In non anemic group, however, no significant change was observed in hemoglobin and hematocrit, meaning that they did not benefit from supplementation.

As of ferritin and transferrin of anemic women, they significantly increased (from 7.5 at recruitment to $53 \mathrm{ng} / \mathrm{mL}$ at term and from 107 to $157 \mathrm{~g} / \mathrm{L}$ at $208-32$ weeks, respectively). In non anemic women notable change occurred only for transferrin $(133-204 \mathrm{~g} / \mathrm{L})$. Serum iron significantly increased $(53-83 \mu \mathrm{g} / \mathrm{dl})$ from recruitment to $28-32$ weeks in anemic women only. So, differences that were significant between groups at recruitment disappeared thereafter. On this basis too, non anemic women did not benefit from supplementation.

Physiologically, hematologic parameters decrease during pregnancy [5]. This is related to hemodilution and fetal demand and is reflected on gradual decline in serum variables such as ferritin, iron and transferrin [5] [16]. That is why the proportion of anemic women tends to rise over pregnancy. The nadir hemoglobin concentration is reached at $24-32$ weeks gestation [5]. After this period, hemoglobin gradually rises till delivery. This could explain why, in our series, improvement of hemoglobin, hematocrit and iron status markers achieved significant level by 28 - 32 weeks, while no effect was observed in non anemic. Restrictions of positive changes induced by iron supplementation in anemic 
Table 8. Association of oxidative stress with nutritional iron intake.

\begin{tabular}{|c|c|c|c|c|c|c|c|c|c|}
\hline \multirow{2}{*}{$\begin{array}{c}\text { Nutritional } \\
\text { iron intake } \\
\text { (mg/day) }\end{array}$} & \multirow{2}{*}{$\begin{array}{c}\text { Period } \\
\text { (weeks of } \\
\text { gestation) }\end{array}$} & \multicolumn{4}{|c|}{ SOD } & \multicolumn{4}{|c|}{ Oxidized LDL } \\
\hline & & Frequency & OR & CI $95 \%$ & $\mathrm{p}$ & Frequency & OR & CI $95 \%$ & $\mathrm{p}$ \\
\hline \multirow{3}{*}{$\geq 18$} & $14-19$ & $56 / 60(93.3 \%)$ & 1.3 & $1.1-1.4$ & 0.424 & $39 / 41(95.1 \%)$ & 1.3 & $0.2-9.5$ & 0.606 \\
\hline & $28-32$ & $48 / 52(92.3 \%)$ & 1.5 & $1.2-1.7$ & 0.235 & $34 / 38(89.5 \%)$ & 2.1 & $1.6-2.6$ & 0.064 \\
\hline & At term & $44 / 48$ (91.7\%) & 1.6 & $1.3-1.9$ & 0.169 & $38 / 42(90.5 \%)$ & 1.8 & $1.5-2.3$ & 0.097 \\
\hline \multirow{3}{*}{$\geq 19$} & $14-19$ & $54 / 60(90 \%)$ & 0.7 & $0.1-6.3$ & 0.604 & $36 / 41(87.8 \%)$ & 0.5 & $0.1-2.6$ & 0.31 \\
\hline & $28-32$ & $46 / 52(88.5 \%)$ & 0.4 & $0.04-3.2$ & 0.323 & $31 / 38(81.6 \%)$ & 2.2 & $1.7-2.8$ & $<0.01$ \\
\hline & At term & $42 / 48(87.5 \%)$ & 0.3 & $0.03-2.5$ & 0.218 & $35 / 42(83.3 \%)$ & 1.9 & $1.5-2.4$ & $<0.02$ \\
\hline \multirow{3}{*}{$\geq 26$} & $14-19$ & $12 / 60(20 \%)$ & 1.7 & $1.2-2.5$ & 0.000 & $8 / 41(19.5 \%)$ & 1.8 & $1.01-3.2$ & $<0.04$ \\
\hline & $28-32$ & $13 / 52(25 \%)$ & 1.3 & $0.9-1.9$ & 0.138 & $8 / 38(21.1 \%)$ & 1.6 & $1.01-2.9$ & $<0.05$ \\
\hline & At term & $11 / 48(22.9 \%)$ & 1.4 & $0.9-2.2$ & 0.071 & $7 / 42(16.7 \%)$ & 2.1 & $1.1-4.01$ & $<0.01$ \\
\hline \multirow{3}{*}{$\geq 27$} & $14-19$ & $11 / 60(18.3 \%)$ & 1.8 & $1.2-2.7$ & 0.000 & $7 / 41(17.1 \%)$ & 1.9 & $1.02-3.6$ & $<0.02$ \\
\hline & $28-32$ & $12 / 52(23.1 \%)$ & 1.3 & $0.9-1.9$ & 0.103 & $7 / 38(18.4 \%)$ & 1.8 & $1.01-3.4$ & $<0.05$ \\
\hline & At term & $11 / 48(22.9 \%)$ & 1.3 & $0.9-2.1$ & 0.126 & $6 / 42(14.3 \%)$ & 2.4 & $1.2-4.8$ & $<0.01$ \\
\hline \multirow{3}{*}{$\geq 30$} & $14-19$ & $7 / 60(11.7 \%)$ & 1.9 & $1.1-3.3$ & 0.001 & $4 / 41(9.8 \%)$ & 2.4 & $1.01-5.6$ & $<0.02$ \\
\hline & $28-32$ & $10 / 52(19.2 \%)$ & 1.1 & $0.7-1.6$ & 0.479 & $3 / 38(7.9 \%)$ & 3 & $1.1-8.3$ & $<0.01$ \\
\hline & At term & $8 / 48(16.7 \%)$ & 1.3 & $0.8-2.1$ & 0.226 & $4 / 42(9.5 \%)$ & 2.4 & $1.02-5.7$ & $<0.02$ \\
\hline \multirow{3}{*}{$\geq 35$} & $14-19$ & $5 / 60(8.3 \%)$ & 1.9 & $1.01-3.7$ & 0.004 & $1 / 41(2.4 \%)$ & 6.9 & $1.1-45.7$ & $<0.01$ \\
\hline & $28-32$ & $8 / 52(15.4 \%)$ & 1.2 & $0.3-4.8$ & 0.578 & $2 / 38(5.3 \%)$ & 3.1 & $1.01-11.2$ & $<0.02$ \\
\hline & At term & $8 / 48(16.7 \%)$ & 1.5 & $0.4-6.4$ & 0.411 & $2 / 42(4.8 \%)$ & 3.5 & $1.01-12.4$ & $<0.01$ \\
\hline \multirow{3}{*}{$\geq 36$} & $14-19$ & $5 / 60(8.3 \%)$ & 1.5 & $0.8-2.8$ & 0.059 & $1 / 41(2.4 \%)$ & 5.5 & $0.9-35.5$ & $<0.01$ \\
\hline & $28-32$ & $7 / 52(13.5 \%)$ & 1.6 & $0.3-8.2$ & 0.463 & $2 / 38(5.3 \%)$ & 2.5 & $0.7-8.6$ & 0.064 \\
\hline & At term & $7 / 48(14.6 \%)$ & 2.1 & $0.4-10.7$ & 0.320 & $2 / 42(4.8 \%)$ & 2.8 & $0.8-9.5$ & $<0.04$ \\
\hline
\end{tabular}

women obviously means that routine administration is useless for non anemic women and might be harmful for them. This aspect is rarely addressed in the literature.

In their review addressing the effects of iron supplementation on hematologic status in pregnancy, Sloan et al. [10] pointed out a finding quite unanimously admitted in the literature: iron supplementation increases hemoglobin levels and ferritin. Amplitude and rate of changes, however, vary according to settings, probably owed to differences in timing of supplementation, dosages of iron, baseline deficiencies, iron absorption conditions, etc.

Despite iron supplementation (dosage and duration of supplementation not specified), Mpawenimana's series In Morocco [16] showed a significant linear regression of ferritin, serum iron and hemoglobin throughout the third trimester. The regression first concerned ferritin and serum iron, secondly the hemoglobin rate. This is in accordance with the fact that hemoglobin will decrease only if reserves drop.

In the Seychelles series (dosage of one tablet containing $200 \mathrm{mg} \mathrm{FeSO}_{4}$ and $0.25 \mathrm{mg}$ folic acid per day) [17] there was no significant difference in ferritin 
concentrations between enrollment and delivery. However, the rate of women with ferritin $<15 \mathrm{ng} / \mathrm{ml}$ increased from $19 \%$ of individuals at recruitment to $24 \%$ at delivery. There was a significant improvement in transferrin levels between enrollment and delivery, a finding reportedly attributed to the increased erythropoiesis iron requirement and iron turnover in the last trimester of pregnancy [17].

Following 8 weeks (20th-36th gestational weeks) of iron supplementation in an Egyptian series of 50 anemic pregnant women $(100 \mathrm{mg}$ of ferrous sulfate twice a day in 25 women and $200 \mathrm{mg}$ ferric hydroxide-IPC once a day in 25 others), Aly et al. [11] found a significant increase $(\mathrm{p}<0.001)$ in hemoglobin and serum ferritin.

Kumar et al [18] assessed serum transferrin and ferritin during pregnancy and their correlation with pregnancy outcome in 56 iron supplemented primigravida (dosage and duration not specified). They found continuous significant rise in serum transferrin and hemoglobin levels in all three trimesters.

This effect of iron supplement is more likely to occur in the presence of iron deficiency. So, the rise is related to initial hematologic status [10]. This can explain why some authors found hemoglobin, serum ferritin and transferrin similar to the presupplementation status [17] [19] [20], presumably meaning that treatment included women in no need of iron supplement. This also emerged from our results clearly showing that, following supplementation, differences that were significant between anemic and non anemic groups at recruitment disappeared thereafter and values of blood parameters tended to be similar for both groups at term. Therefore, non anemic women did not benefit from supplementation.

The effect of iron supplementation has been found dose (more than 91 $\mathrm{mg} /$ day) along with duration (11 - 13 weeks) dependent. With $60 \mathrm{mg}$ of iron (ferrous sulfate)/day, Falahi et al. [21], in Iran, got a significant difference of hemoglobin and ferritin rates between supplemented women and placebos. Nevertheless, a daily supplement of $30-40 \mathrm{mg}$ iron during the third trimester has been suggested as adequate to maintain satisfactory hematological status during pregnancy [15] [22].

Eskeland et al. [23] found that even a smaller daily dose of $27 \mathrm{mg}$ iron has a demonstrable positive effect on iron status. In a placebo-controlled Danish dose-response study, Milman et al. [24] found no significant differences in the serum ferritin concentrations in women taking 40,60 , or $80 \mathrm{mg}$ of ferrous iron daily. A daily dose of $40 \mathrm{mg}$ of ferrous iron thus appeared to be adequate to prevent iron deficiency anemia in more than $95 \%$ of the women. Although the role of antioxidant vitamins in facing oxidative stress is widely admitted [25] no study claimed better responses with conjunction of antioxidant vitamins with iron.

In our series, women with anemia $(\mathrm{Hb}<10 \mathrm{~g} \%)$ received $320 \mathrm{mg} /$ day of ferric ammonium citrate for curative while the others received $160 \mathrm{mg} /$ day for preventive purposes. These huge dosages in comparison with the above references are 
still common in our country without obvious scientific basis. To support them, one could advocate limiting conditions of iron absorption in our setting such as the predominantly non-heminic nature of alimentary iron, intestinal parasitosis and chelating substances contained in food (phytates, phosphates, tannins, fibers and calcium salts) [13] [14]. Even though, this represents a threat for oxidative status of individuals as we discuss it later.

With respect to oxidative status during pregnancy with or without any supplementation, very few studies addressed its own potential variation throughout normal gestation. The evaluation is usually carried out either early [26] or at any gestational age [9] [11] [27] [28]. Based on considerations put forth by Liochev \&. Friedovich [29], as the placenta matures, the development of its vascularization induces an oxygen-rich environment and its abundant mitochondrial mass favors the production of reactive oxygen species, which increases free iron liberated from iron-sulfur clusters [1]. Furthermore, due to physiological decline of iron status it might be assumed that baseline oxidative status worsens throughout pregnancy [30]. No study, however, has confirmed this in healthy women with uncomplicated pregnancy.

So far, the only data available on intrinsic changes of oxidative status throughout normal pregnancy deal with the placenta and come from Basu et al. [31] who investigated women with uncomplicated pregnancies. They found an increased oxidative stress in the placenta (high total antioxidant capacity and lipid peroxide levels) in the early phase. With the progression of pregnancy, placental antioxidant protective mechanisms increased and lipid per-oxidation markers decreased. This phenomenon was related to hypoxic environment of the placenta in early pregnancy and physiological decrease in placental oxidative stress thereafter. Although there is no evidence of the same profile with maternal blood, findings of this study can serve as reference to support the decrease of oxidative stress as pregnancy progresses [31].

What about changes induced by iron supplementation on oxidative status? Given that iron deficiency is associated with oxidative stress [2] [11] [30], may supplementation trigger antioxidant capacity of individuals? In our study, SOD values decreased alongside supplementation, although no significant difference was observed trimester wise. Since SOD represents antioxidant activity, this would mean an increased vulnerability to oxidative stress. At 28 - 32 weeks, anemic and non anemic women were still different (SOD higher in anemic). For oxidized LDL, both in anemic and non anemic women, values significantly increased from recruitment to term, values remaining significantly higher in anemic women. These findings mean a continuous increase in oxidative threat following iron administration, regardless of difference in doses of each group, although significant associations with oxidative stress appeared only for oxidized LDL after $\geq 20$ weeks of supplementation (OR = 7.04; CI 95\%: 2.5 - 19.7; p < $0.001)$.

In Estonia, Rehema et al. [8] measured the effect of ferrous iron therapy (18/36 mg of ferrous aspartate daily, +Fe group, $n=13$ and no-treatment group, 
$\mathrm{n}=6$ ) on indices of oxidative stress in 19 healthy pregnant women with borderline anemia. After a study period of 4 weeks they observed a significant increase of both lipid peroxidation and antioxidative defense (mostly the glutathione system) but without significant difference between groups. The authors rose caution on their conclusion due to smallness of their sample.

In the Egyptian study, Aly et al. [11] observed an increased oxidative stress with concurrent high levels of total antioxidant capacity and malondialdehyde (one of the final products of polyunsaturated fatty acids peroxidation in the cells).

Kurtoglu et al. [31] measured the effect of iron supplementation on antioxidant status in 63 iron-deficient patients after $6 \mathrm{wk}$ of treatment. A significant decrease of oxidative stress was observed. The levels of catalase, SOD and glutathione peroxidase increased $(\mathrm{p}<0.05)$ and showed no more significant differences till the end of the study, at the time when body iron stores are saturated.

So, while oxidative stress decreases itself as pregnancy progresses [31], anemia makes it increase [2] [30]. Therefore, iron supplementation is supposed to reverse this tendency and restore the balance [9] [10] [32]. Our study showed the contrary. Other authors who reported increase of oxidant activity after supplementation [8] [11] [30] observed increase of levels in both lipid peroxidation and antioxidative defense, instead of increased oxidants allied with decreased antioxidants or vice versa. Our own study [2] also found a baseline status with concurrent increase of levels in both lipid peroxidation and antioxidative defense. The definite answers pertaining to these apparently conflicting aspects are probably linked to variations of situations encountered by searchers. Indeed, purpose of studies (curative or preventive), iron doses, period and duration of treatment, nutritional intake and intestinal absorption conditions differ with settings. Most studies simply fail to mention these issues. In the current study we clearly observed that the lowest duration of iron replacement to be associated with oxidative stress was 20 weeks. Regardless of pathologic situations, many other confounding factors responsible for changes in oxidative status profiles following iron administration can be found in environment and season [33], psychosocial status [34], intestinal parasites and malaria [13] [14] [30] [35] etc.

Actually, after correcting anemia by achieving optimal hemoglobin levels and building up iron stores (ferritin levels), such as we got it in our study, total antioxidant capacity might be boosted. In our series, this probably occurred at 28 32 weeks, after 11 - 12 weeks of supplementation (Table 2). Furthering iron supplementation thus favored oxidative stress, although significant associations of duration of iron replacement with oxidative stress appeared only for oxidized LDL (OR = 7.04; CI 95\%: 2.5 - 19.7; p < 0.001) after $\geq 20$ weeks of supplementation (Table 6). This concerns mostly anemic women for which iron was provided for longer time.

So, it can be suggested that additional oral iron in so replete patients remains unabsorbed. Its pro-oxidant properties are likely to induce production of free radical oxygen species and thereby impact oxidative status that was observed till 
term. Iron supplement is to be considered together with nutritional intake mostly providing non-heminic iron and whose significant association with oxidative stress was found at $26 \mathrm{mg} /$ day for SOD (OR $=1.7$; CI 95\%: $1.2-2.5 ; \mathrm{p}<$ $0.001)$ and oxidized LDL (OR = 1.8; CI 95\%: $1.01-3.2 ; \mathrm{p}<0.04)$ at recruitment and even at $19 \mathrm{mg} /$ day by $28-32$ weeks of gestation for oxidized LDL $(\mathrm{OR}=2.2$; CI 95\%: 1.7 - 2.8; $\mathrm{p}<0.01$ ). Lund et al. [36] clearly demonstrated a steady increase of fecal iron with $40 \%$ increased production of free radicals at colon after iron administration.

\section{Conclusions}

Iron supplementation improved iron status in anemic women, not in non anemic. Differences that were significant between groups at recruitment disappeared by 28 - 32 weeks. Non anemic women were not in need of iron supplementation. SOD decreased and LDL values increased alongside supplementation. This represents a growing oxidant threat for both anemic and non anemic women. This could be related to iron overload and/or unabsorbed iron.

Doses of iron should be decreased (at least halved) and duration of treatment restricted to not more than 12 weeks.

\section{Limitation and Strength of the Study}

Study limitation mainly relates to smallness of the study sample, a factor already allegedly owed to cost of an own funded study. The strength of the study comes first from the scarcity of studies addressing both iron status and oxidative status during pregnancy in Sub-Saharan Africa, secondly from actual routine nature of the supplementation, involving preventive and curative concerns. Attention paid on this later aspect helps question harmful influence of iron supplements in women who are not in need of it. Furthermore, our study included many confounding factors such as dietary and nutritional details accountable for in understanding of some conflicting findings.

\section{Author's Contributions}

MMA is the principal investigator, participated in designing the study and was actively involved in data collection and statistical calculations. TUB generated and designed the study. He drafted the first manuscript. MMR participated in designing the study and analyzing the results. All authors contributed in preparing the final manuscript.

\section{Conflicts of Interest}

The authors declare no conflicts of interest regarding the publication of this paper.

\section{References}

[1] Friedrisch, J.R. and Friedrisch, B.K. (2017) Prophylactic Iron Supplementation in 
Pregnancy: A Controversial Issue. Biochemistry Insight, 10, 1-8. https://doi.org/10.1177/1178626417737738

[2] Mbangama, M.A., Tandu-Umba, B. and Mbungu, M.R. (2018) Baseline Iron Status and Indices of Oxidative Stress in a Cohort of Pregnant Women in Kinshasa, DR Congo. Open Journal of Obstetrics and Gynecology, 8, 1476-1486.

https://doi.org/10.4236/ojog.2018.814149

[3] Achebe, M.M. and Gafter-Gvili, A. (2017) How I Treat Anemia in Pregnancy: Iron, Cobalamin and Folate. Blood, 129, 940-949. https://doi.org/10.1182/blood-2016-08-672246

[4] Koenig, M.D., Tussing-Humphreys, L., Day, J., Cadwell, B. and Nemeth, E. (2014) Hepcidin and Iron Homeostasis during Pregnancy. Nutrients, 6, 3062-3083. https://doi.org/10.3390/nu6083062

[5] Milman, N. (2006) Iron and Pregnancy: A Delicate Balance. Annals of Hematology, 85, 559-565. https://doi.org/10.1007/s00277-006-0108-2

[6] Casanueva, E. and Viteri, F.E. (2003) Iron and Oxidative Stress in Pregnancy. The Journal of Nutrition, 133, 1700S-1708S. https://doi.org/10.1093/jn/133.5.1700S

[7] Tiwari, A.K.M., Mahdi, A.A., Chandyan, S., Godbole, M.M., Jaiswar, S.P., Srivastava, V.K. and Negi, M.P. (2011) Oral Iron Supplementation Leads to Oxidative Imbalance in Anemic Women: A Prospective Study. Clinical Nutrition, 30, 188-193. https://doi.org/10.1016/j.clnu.2010.08.001

[8] Rehema, A., Zilmer, K. Klaar, U., Karro, H., Kullisaar, T. and Zilmer, M. (2004) Ferrous Iron Administration during Pregnancy and Adaptational Oxidative Stress (Pilot Study). Medicina (Kaunas), 40, 547-552.

[9] Lymperaki, E., Tsikopoulos, A., Makedou, K., Paliogianni, E., Kiriazi, L., Charisi, C. and Vagdatli, E. (2015) Impact of Iron and Folic Acid Supplementation on Oxidative Stress during Pregnancy. Journal of Obstetrics and Gynaecology, 35, 803-806. https://doi.org/10.3109/01443615.2015.1011102

[10] Sloan, N.L., Jordan, E. and Winikoff, B. (2002) Effects of Iron Supplementation on Maternal Hematologic Status in Pregnancy. American Journal of Public Health, 92, 288-293. https://doi.org/10.2105/AJPH.92.2.288

[11] Aly, S.S., Fayed, H.M., Ahmed, S.S., Abdella, A.H., Tamam, A.A.E. and Mohmmed, N.A. (2016) Effects of Oral Iron (Ferrous Versus Ferric) Supplementation Onoxidative Stress and Antioxidant Status in Pregnant Women with Iron Deficiency: Controlled Trial. The Egyptian Journal of Haematology, 41, 31-41. https://doi.org/10.4103/1110-1067.186392

[12] Mbangama, M.A, Tandu-Umba, B. and Mbungu, M.R. (2018) Rationale of a Cohort Study on Risk of Obstetrical Outcomes Associated with Iron Supplementation during Pregnancy. Open Journal of Obstetrics and Gynecology, 8, 598-609. https://doi.org/10.4236/ojog.2018.86066

[13] Tandu-Umba, N.F.B. and Mputu Lobota, A. (1995) Geophagia in Pregnant Women in Kinshasa, Zaïre. Journal Africain des Sciences Biomédicales, 2, 34.

[14] Tandu-Umba, N.F.B. (1995) Promotion de la bio-disponibilité du fer alimentaire au sein de la population obstétricale de Kinshasa. Médecine et Nutrition, 31, 19-22.

[15] Milman, N. (2006) Iron Prophylaxis in Pregnancy-General or Individual and in Which Dose? Annals of Hematology, 85, 821-828. https://doi.org/10.1007/s00277-006-0145-x

[16] Mpawenimana, S. (2010) Suivi de l'évolution du statut martial au cours de la grossesse: Etude prospective réalisée à l'Hôpital Militaire d'instruction Mohamed V de 
Rabat. Thèse de doctorat en pharmacie, Faculté de Médecine et de Pharmacie, Université Mohammed V, Rabat.

[17] Duffy, E.M., Bonham, M.P., Wallace, J.M.W., Chang, C.K., Robson, P.J., Myers, G.J., Davidson, P.W., Clarkson, T.W., Shamlaye, C.F. and Strain, J.J. (2010) Iron Status in Pregnant Women in the Republic of Seychelles. Public Health Nutrition, 3 , 331-337. https://doi.org/10.1017/S1368980009991054

[18] Kumar, S., Dubey, N. and Khare, R. (2017) Study of Serum Transferrin and Serum Ferritin during Pregnancy and Their Correlation with Pregnancy Outcome. International Journal of Medical Science and Public Health, 6, 118-122. https://doi.org/10.5455/ijmsph.2017.04072016570

[19] Yip, R. (1996) Iron Supplementation during Pregnancy: Is It Effective? American Journal of Clinical Nutrition, 63, 853-855. https://doi.org/10.1093/ajcn/63.6.853

[20] Nair, K.M., Bhaskaram, P., Balakrishna, N., Ravinder, P. and Sesikeran, B. (2004) Response of Hemoglobin, Serum Ferritin and Serum Transferrin Receptor during Iron Supplementation in Pregnancy: A Prospective Study. Nutrition, 20, 896-899. https://doi.org/10.1016/j.nut.2004.06.011

[21] Falahi, E., Akbari, S., Ebrahimzade, F. and Gargari, B.P. (2011) Impact of Iron Supplementation of Healthy Pregnant Women on Maternal Iron Status and Birth Outcome. Food and Nutrition Bulletin, 32, 213-217. https://doi.org/10.1177/156482651103200305

[22] Nair, K.M., Dripta, R.C. and Konapur, A. (2018) Appropriate Doses of Iron for Treatment of Anemia Amongst. Indian Journal of Community Health, 30, 39-53.

[23] Eskeland, B., Malterud, K., Ulvik, R.J. and Hunskaar, S. (1997) Iron Supplementation in Pregnancy: Is Less Enough? A Randomized, Placebo Controlled Trial of Low Dose Iron Supplementation with and without Heme Iron. Acta Obstetricia et Gynecologica Scandinavica, 76, 822-828. https://doi.org/10.3109/00016349709024359

[24] Milman, N., Bergholt, T., Eriksen, L., Byg, K.E., Graudal, N., Pedersen, P. and Hertz, J. (2005) Iron Prophylaxis during Pregnancy-How Much Iron Is Needed? A Randomized Dose-Response Study of 20-80 mg Ferrous Iron Daily in Pregnant Women. Acta Obstetricia et Gynecologica Scandinavica, 84, 238-247. https://doi.org/10.1111/j.0001-6349.2005.00610.x

[25] Umar Imam, M., Zhang, S., Ma, J., Wang, H. and Wang, F. (2017) Antioxidants Mediate Both Iron Homeostasis and Oxidative Stress. Nutrients, 9, 671. https://doi.org/10.3390/nu9070671

[26] Shastri, L., Pammal, R.S., Mani, I., Thomas, T. and Kurpad, A.V. (2016) Oxidative Stress during Early Pregnancy and Birth Outcomes. Public Health Nutrition, 19, 3210-3215. https://doi.org/10.1017/S1368980016001191

[27] Aslan, M., Horoz, M. and Çelik, H. (2011) Evaluation of Oxidative Status in Iron Deficiency Anemia through Total Antioxidant Capacity Measured Using an Automated Method. Turkisch Journal of Hematology, 28, 42-46. https://doi.org/10.5152/tjh.2011.04

[28] Imene, B. (2013) Détermination du statut oxydant/antioxydant au cours de la grossesse obèse avec ou sans complications (Diabète, Hypertension) dans la région de Tlemcen. Mémoire en vue de l'obtention du diplôme de Master en Biologie, Université Abou Bekr Belkaïd Tlemcen.

[29] Liochev, S.I. and Friedovich, I. (1997) How Does Superoxide Dismutase Protect against Tumor Necrosis Factor: A Hypothesis Informed by Effect of Superoxide on "Free" Iron. Free Radical Biology and Medicine, 23, 668-671. 
https://doi.org/10.1016/S0891-5849(97)00060-9

[30] Kumar, N., Chandhiok, N., Dhillon, B.S. and Kumar, P. (2009) Role of Oxidative Stress While Controlling Iron Deficiency Anemia during Pregnancy-Indian Scenario. Indian Journal of Clinical Biochemistry, 24, 5-14.

https://doi.org/10.1007/s12291-009-0002-5

[31] Basu, J., Bendek, B., Agamasu, E., Salafia, C.M., Mishra, A., Benfield, N., Patel, R. and Mikhail, M. (2015) Placental Oxidative Status throughout Normal Gestation in Women with Uncomplicated Pregnancies. Obstetrics and Gynecology International, 2015, Article ID: 276095. https://doi.org/10.1155/2015/276095

[32] Kurtoglu, E., Ugur, A., Baltaci, A.K. and Undar, L. (2003) Effect of Iron Supplementation on Oxidative Stress and Antioxidant Status in Iron Deficiency Anemia. Biological Trace Element Research, 96, 117-123.

https://doi.org/10.1385/BTER:96:1-3:117

[33] Elongi, M.J.P., Scheers, H., Tandu-Umba, B., Haufroid, V., Buassa-bu-Tsumbu, B., Verdonck, F., Spitz, B. and Nemery, B. (2016) High and Season-Dependent Urinary Excretion of Toxic Metals in Women with Preeclampsia. A Case-Control Study in Kinshasa, DR Congo. Environmental Health, 15, 48.

[34] Tandu-Umba, B., Dedetemo, K.D. and Mananga, L.G. (2014) Maternal Stress and Pregnancy Outcomes. Open Journal of Obstetrics and Gynecology, 4, 361-370. https://doi.org/10.4236/ojog.2014.47054

[35] Ifoue, S.H.T., Mofor, C.T., Gouado, I., Teto, G., Asonganyi, T. and Zollo, P.H.A. (2009) Evaluation of Oxidative Stress and Antioxidant Status of Pregnant Women Suffering from Malaria in Cameroon. Indian Journal of Clinical Biochemistry, 24, 288-293. https://doi.org/10.1007/s12291-009-0054-6

[36] Lund, E.K., Wharf, S.G., Fairweather-Tait, S.J. and Johnson, I.T. (2003) Oral Ferrous Sulfate Supplements Increase the Free Radical Generating Capacity of Feces from Healthy Volunteers. The American Journal of Clinical Nutrition, 78, 498. 